\title{
THERMALLY STIMULATED ELECTRON EMISSION FROM K-Bi-GICs
}

\author{
J. Chrzanowski \\ Institute of Experimental Physics, University of Wrocław \\ Max Born Sq. 9, 50-204 Wrocław, Poland \\ R. KLIMKIEWICZ \\ Institute of Low Temperature and Structure Research \\ Polish Academy of Sciences, PO Box 937, 50-950 Wroclaw, Poland \\ AND A.W. MoraWsKI \\ Institute of Inorganic Chemical Technology \\ Technical University of Szczecin, Pułaskiego 10, 70-322 Szczecin, Poland
}

(Received September 16, 1997; revised version December 1, 1997)

The results of the investigations of thermally stimulated electron emission from graphite submitted to intercalation of $\mathrm{K}-\mathrm{Bi}$ alloy, in the temperature range of $100-400 \mathrm{~K}$, are presented. Total and partial pressure in the measuring chamber in relation to the temperature of the sample are also reported. The insertion of a $\mathrm{K}-\mathrm{Bi}$ alloy into the interlayers of graphite considerably changes thermally stimulated electron emission properties. The emission images $I(T)$ depend on the content of intercalate and the emission maxima obtained at temperatures of about 170 and $210 \mathrm{~K}$ are conditioned by the desorption of a gas while the total pressure above the different samples is of the same order. The desorption of gases at a temperature over $250 \mathrm{~K}$ does not cause any increase in the electron emission.

PACS numbers: $71.20 . \mathrm{Tx}, 79.75 . \mathrm{g}, 79.20 . \mathrm{Kz}$

\section{Introduction}

To characterize the thermally stimulated electron emission (TSEE) phenomenon [1-3] it is convenient to divide it into three stages: excitation of the sample, creation of free electrons during stimulation, and electron transport towards the surface of the sample and their transfer into vacuum.

The irradiation of the samples by electron beam at $100 \mathrm{~K}$ prior to TSEE measurements leads to the partial localization of electrons in the electron traps 
- the emission centers related, for example, with structural defects. In the next stage, during thermostimulation process, free electrons in the conduction band are created. The energy supplied to the sample during heating process can be sufficient for ionization of the electron traps and transfer of these delocalized electrons into conduction band. The subsequent stage is the electron transport towards the surface and their escaping into vacuum. The intensity of electron current is proportional to the concentration of active emission centers. Thus, the concentration of defects-emission centers can be estimated as the area under the TSEE curve.

The TSEE method is suitable for the study of systems in which the electronhole centers are created, for example, in the case of graphite intercalates. The amphoteric character of graphite leads to the creation of compounds of electrondonor-acceptor (EDA)-type. In binary graphite intercalation compounds (GICs) of acceptor-type there appears the transfer of the $\pi$ electrons of graphite to the valence band, and the produced GICs are $p$-type conductors, e.g. graphite $\mathrm{FeCl}_{3}$. In intercalates of donor-type, e.g. K-GICs, the donor electrons are carried to the $\pi$ graphite system, creating conductors of $n$-type. In ternary GICs, e.g. $\mathrm{K}-\mathrm{Hg}-\mathrm{GICs}, \mathrm{K}-\mathrm{Bi}-\mathrm{GICs}$, the transfer of electrons and creating of EDA system are more complicated. Up to now the problem of electron structure and the electron transfer direction are still debatable. In the case of $\mathrm{KHgC}_{4}$ and of $\mathrm{KHgC}_{6}$ with the use of X-ray photoelectron spectroscopy (XPS) [4] method, there was noticed a higher charge of $\mathrm{K} 4 s$ electrons than in $\mathrm{KC}_{8}$, a higher asymmetry of this charge caused by the increase in the density of conductivity electrons occupying valence orbitals of ionized central atom $(\mathrm{Hg})$ or of hybridized system formed from the orbitals of those atoms. The author of Ref. [4] suggests that $s$ electrons of the central atom are responsible for superconductivity at low temperatures. Herold [5-8] suggests the following arrangement of the electrons for this type of compounds: $\mathrm{M}^{+} \mathrm{C}^{-} \mathrm{M}^{+} \mathrm{Hg}^{-} \mathrm{M}^{+} \mathrm{C}^{-} \mathrm{M}^{+} \ldots$ (where $\mathrm{M}$ - alkali metal), i.e. the direction of the electron transfer is from the alkali metal through graphite to the central atom.

There is no information in the available literature concerning the study of electron emission from intercalate materials at low temperatures. It was, therefore, the aim of this study. We have been interested to determine the electron emission properties of $\mathrm{K}-\mathrm{Bi}-\mathrm{C}$ intercalate, in the temperature range from 100 to $400 \mathrm{~K}$, during heating as well as during cooling the sample.

Intercalation compounds of graphite have been studied lately owing to the possibility of designs of crystallographic and electron system [9, 10]. The study of the electron emission activity of these intercalate samples can be useful for the control of technology of intercalate samples preparation. The studies can also contribute to the explanation of catalytic activity $[11,12]$ and conductivity of these materials.

\section{Experimental and results}

Three types of samples were examined: pure graphite and two samples with different contents of intercalate, in the work described as $\mathbf{H}$ and L. Sample $H$ contained enriched fraction of intercalate $\mathrm{K}-\mathrm{Bi}$-graphite, of $\mathrm{K}_{4.7} \mathrm{BiC}_{29.5}$ total composition. The main phases in that sample were: a mixture of $\mathrm{K}-\mathrm{Bi}-\mathrm{GICs}$ with three different identity periods (1425 pm, $1381 \mathrm{pm}$ and $991 \mathrm{pm}$ ) and graphite (ca. 5\%). 
Sample L contained free graphite (over $90 \%$ ) and a small amount of $\mathrm{K}-\mathrm{Bi}-\mathrm{GICs}$ of different stages (from the second stage upwards). The preparation of the studied samples consisted in heating the formerly prepared $\mathrm{K}$-Bi-alloy with powdered Sri Lanka graphite. During the preparation, the samples were heated up to the temperature of $730 \mathrm{~K}$ in the $\mathrm{N}_{2}$ atmosphere. Pure graphite was treated in the same manner. The studied samples were formed into tablets measuring: diameter $-20 \mathrm{~mm}$ and thickness $-2 \mathrm{~mm}$.

For TSEE measurements the measuring chamber was built. The measuring stand enabled us to produce vacuum of the order of $10^{-7} \mathrm{~Pa}$. The measuring head, made of molybdenum glass contained: detection system (electron multiplier of the tube-type, grid), electron gun, monopole mass spectrometer (UFM) and Bayard Alpert's head. The body as well as the measuring table were made of stainless steel $1 \mathrm{H} 18 \mathrm{~N} 9 \mathrm{~T}$. To measure the temperature of the sample, two copper-constantan differential thermocouples were used. The fiducial temperature in emission measurement was the sample surface temperature.

Before the temperature stimulation, the samples were excited by electron beam with the energy of $300 \mathrm{eV}\left(I_{\mathrm{e}}=5 \mu \mathrm{A}\right)$, at a temperature of about $100 \mathrm{~K}$ for $30 \mathrm{~s}$. Next, the temperature of the sample was changed from $100 \mathrm{~K}$ to $400 \mathrm{~K}$ (the average rate of heating $-10 \mathrm{~K} / \mathrm{min}$.), once more irradiated by electron beam at a temperature of about $400 \mathrm{~K}$, and then cooled to $100 \mathrm{~K}$ (the average rate of cooling $9 \mathrm{~K} / \mathrm{min}$.). After each cycle of this pattern (100-400-100 K) the sample was bombarded again and the measuring cycle was repeated.

A typical TSEE curve $I(T)$ obtained for sample $\mathrm{H}$ is presented in Fig. 1. During heating the sample we can observe a slow decrease in the electron emission intensity and two maxima at 170 and $210 \mathrm{~K}$. During cooling no definite maxima on $I(T)$ curves have been observed, and electron emission intensity was considerable lower (an order of magnitude) than during heating (Fig. 2).

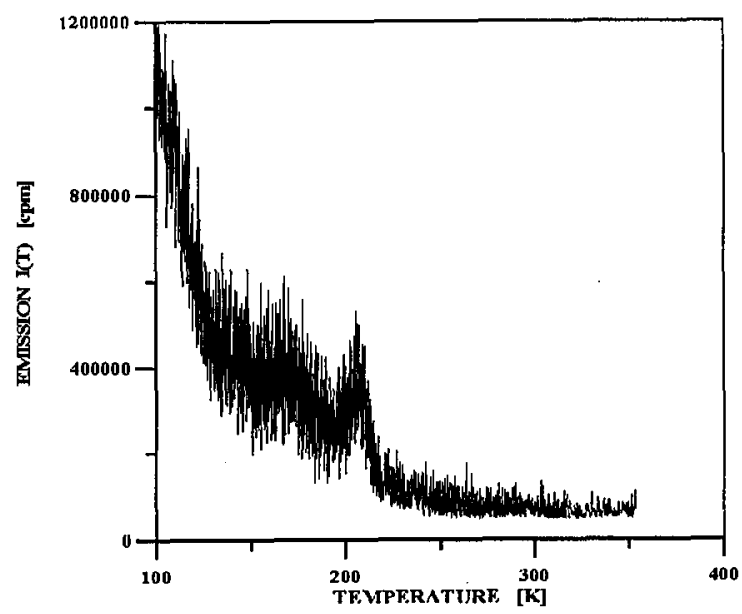

Fig. 1. Electron emission intensity as a function of temperature recorded during heating H-type sample. 


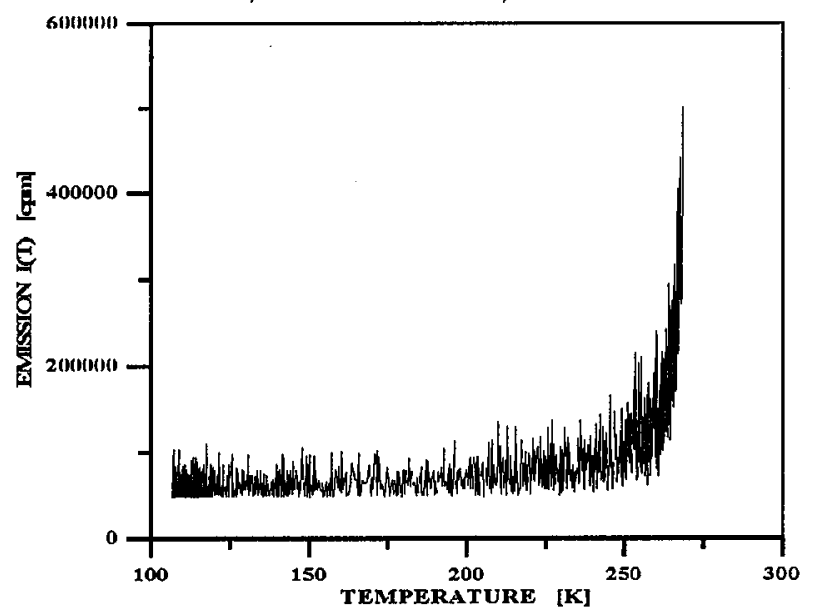

Fig. 2. Electron emission intensity as a function of temperature recorded during cooling H-type sample.

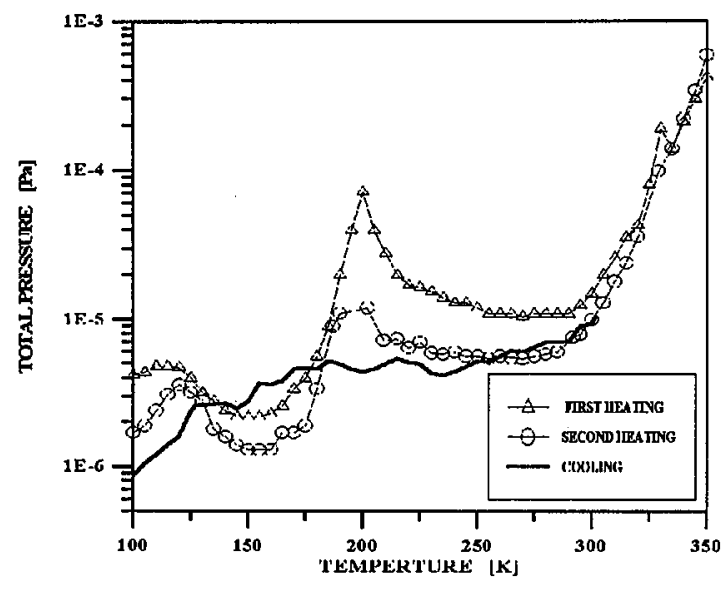

Fig. 3. Total pressure in the measuring chamber during heating and cooling H-type sample.

The change in total pressure in the apparatus during heating and cooling is shown in Fig. 3. During temperature increasing, an increase in total pressure in the measuring chamber is observable. At temperatures of about 120 and $200 \mathrm{~K}$ there appear maxima of pressure and at $300 \mathrm{~K}$ the constant increase in pressure is observed.

During cooling the sample there is a slow decrease in pressure in the measuring chamber. The emission image obtained during heating L-type samples is shown in Fig. 4. Emission maxima being several times lower appear at temperatures of about $170 \mathrm{~K}$ and $200 \mathrm{~K}$.

The changes of the pressure in the measuring chamber during heating and cooling samples of this type are presented in Fig. 5. At temperatures of 130 and 


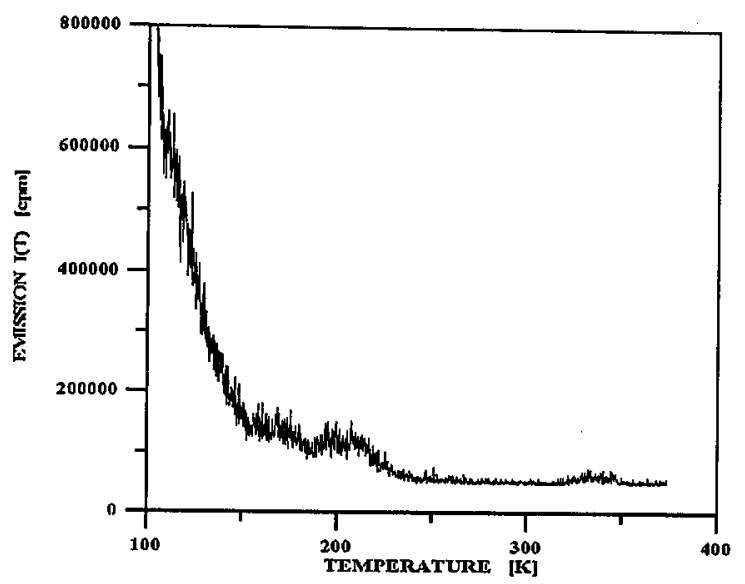

Fig. 4. Electron emission intensity as a function of temperature recorded during heating L-type sample.

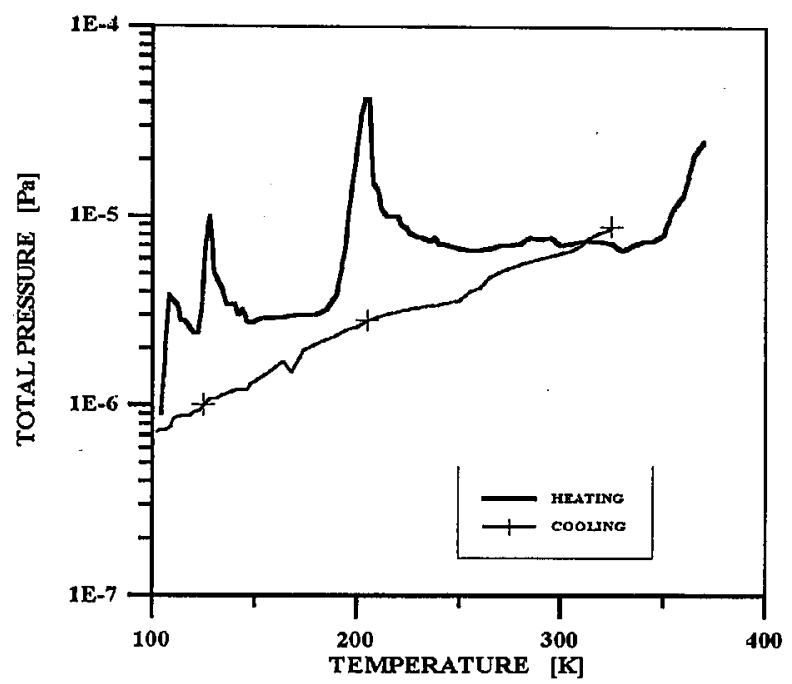

Fig. 5. Total pressure in the measuring chamber during heating and cooling L-type sample.

$200 \mathrm{~K}$ there appears a considerable increase in pressure, and from $350 \mathrm{~K}$ its constant increase. The multiple repetition of the consecutive measuring cycles caused the consistent diminution of the quantity of desorbed gases whereas the electron emission maxima, its position and dimension, remained much the same.

The measurements of the graphite sample proved that during consecutive heating-cooling process there is a weak, temperature independent electron emission. TSEE measurements did not show any emission maxima in the studied temperature range.

From the partial pressure measurements with the use of the monopole mass spectrometer we can state that the intercalate samples content a considerable 
amount of $\mathrm{H}_{2} \mathrm{O}, \mathrm{CO}$ and $\mathrm{CO}_{2}$ molecules, which is the feature of the materials of this type.

Comparing Figs. 1 and 4, it can be noticed that the increase in the amount of intercalate in the sample causes a higher electron emission; whereas the comparison of Figs. 3 and 5 shows that the pressure of emitted gases is of the same range so it is independent of the amount of intercalate. Thus, the achieved electron emission is a sum and can depend on the contribution of the electrons from ionized gases as well as the electrons emitted from the sample.

Studying the influence of temperature on conductivity of monocrystal compounds M-Bi-GICs (where M-alkali metal), Lagrange et al. [9] ascertained the presence of "mixed 'metallic' and thermally activated $c$-axis conductivity" at a temperature of about $295 \mathrm{~K}$ for stage 1 . The temperature of transition from metallic to thermally activated conductivity diminishes together with the decrease in amount of $\mathrm{M}-\mathrm{Bi}$ in graphite. Our research confirms their observations.

\section{Conclusions}

Samples with different contents of intercalate were used. The emission maxima obtained for H-type sample (considerable amount of intercalate can be the reason for the increased amount of emission centers in the sample) at temperatures of about 170 and $200 \mathrm{~K}$ can be connected with the desorption of a definite gas from the sample, the increase in the pressure at this temperature range being the evidence for this fact. A similar emission image is observable for L-type sample but the emission intensity is considerably lower than for sample H. Taking into account that the measurements of pure graphite do not show any emission centers at the studied temperature range, it can be said that the insertion of a $\mathrm{K}-\mathrm{Bi}$ alloy into the interlayers of the graphite and creating $\mathrm{K}-\mathrm{Bi}-\mathrm{GICs}$ considerably changes the emission properties of the sample. The electron emission intensity depends on the intercalate content.

The measurements of the partial pressure show that the pressure of the desorbed gas does not depend on the content of the intercalate.

The desorption of gases from the sample at temperatures over $300 \mathrm{~K}$ does not cause any increase in the emission in the studied samples, which leads to the assumption that gases desorbed in lower temperature were situated at other centres than gases desorbed over $300 \mathrm{~K}$.

From the observed electron emission images it can be assumed that changes of the emission activity of samples were caused by structure reconstruction and creation of new electron-donor centers in the $170-200 \mathrm{~K}$ range. This reconstruction can facilitate the desorption of gas from the sample as well as can cause liberation of electrons from emission centers and ionization of released gases.

The emission image can constitute the characteristics of these materials describing the participation of metallic and thermally activated conductivity. It seems to be necessary to perform supplementary research on samples of this type in respect of the emission. It can be useful for the control of technology of receiving materials of high conductivity. 


\section{Acknowledgment}

This work was supported by Wrocław University grant No. 2016/W/IFD/96.

\section{References}

[1] H.J. Fitting, in: Proc. 6th Int. Symp. EEE and Applications, Ahrenshoop, Eds. H. Glaefeke, W. Wild, Univ. Rostock, Rostock 1979, p. 23.

[2] V.S. Kortov, A.I. Slesarev, Fiz. Tverd. Tela 17, 926 (1975).

[3] V.S. Kortov, in: Proc. 9th Intern. Symp. Exoelectron Emission and Applications, Wrocław, Eds. B. Sujak, A. Scharmann, K. Gieroszyńska, A. Gieroszyński, Institute of Physics, University of Giessen (Germany) 1988, p. 5.

[4] S.B. DiCenzo, Synth. Metals 12, 251 (1985).

[5] A. Herold, G. Furdin, D. Guerard, L. Hachim, N.-E. Nadi, R. Vangelisti, Ann. Phys. (Paris) 11, (2) 3 (1986).

[6] A. Herold, G. Furdin, D. Guerard, L. Hachim, M. Lelaurain, N.-E. Nadi, R. Vangelisti, Synth. Metals 12, 11 (1985).

[7] A. Herold, in: Physics of Intercalation Compounds, Proc. Inter. Conf., Trieste (Italy) 1981, Eds. L. Pietronero, E. Tasatti, Springer-Verlag, Berlin-Heidelberg-New York 1981, p. 7.

[8] A. Herold, D. Billaud, D. Guerard, P. Lagrange, M. El Makrini, Physica B 105, 253 (1981).

[9] P. Lagrange, A. Bendriss-Rerhrhaye, J.F. Mareche, E. McRae, Synth. Metals 12, 201 (1985).

[10] P. Lagrange, A. Bendriss-Rerhrhaye, Carbon 26, 283 (1988).

[11] R. Klimkiewicz, A.W. Morawski, W. Miśta, J. Catal. 144, 627 (1993).

[12] J. Rakoczy, R. Klimkiewicz, A.W. Morawski, J. Phys. Chem. Sol. 57, 805 (1996). 